\title{
Photo-controlled deformable mirror for wavefront shaping
}

\author{
Frederic Zamkotsian ${ }^{1}$, Lorenzo Cabona ${ }^{2}$, Edoardo Redaelli ${ }^{2}$, Giorgio Pariani ${ }^{2}$, \\ Patrick Lanzoni ${ }^{1}$, Andrea Bianco ${ }^{2}$ \\ ${ }^{1}$ Aix Marseille Université, CNRS, CNES, LAM (Laboratoire d'Astrophysique de Marseille), 13388, Marseille, France \\ ${ }^{2}$ INAF - Osservatorio Astronomico di Brera, Via Bianchi 46, 23807 Merate, Italy \\ e-mail: frederic.zamkotsian@lam.fr
}

\begin{abstract}
Wavefront shaping is of main interest in the field of Adaptive Optics for Astronomy, wide-field imaging in Microscopy and live cells/tissues imaging in Biology.

We propose an innovative technology for a new type of membrane deformable mirror, made by coupling a reflective polymeric membrane with a monolithic non-pixelated photoconductive substrate. The device is called continuous photocontrolled deformable mirror (PCDM), actuated by sending a light flux with given shape and intensity distribution, on the back side of the photoconductor, opposite to the reflective side. Unlike the other actuation mechanisms, this allows to obtain a continuous actuation field, without the typical segmented actuators pattern. Furthermore, it leads to a strong simplification to the driving electronics, for example by eliminating hundreds of cables used for the deformation control. Fundamental is the role played by the photo-excitation dynamics of the photoconductive material, in fact the deformation is obtained through the electrostatic pressure exerted by the photo-generated charge carriers, on the thin reflective membrane.

We have developed a device with a single crystalline semiconductor photoconductor wafer, either Silicon or Cadmium Sulfide. The actuation is controlled by a light pattern modulated by a DMD (Digital Micromirror Device) chip, generating a reprogrammable actuator pattern projected on the photoconductor. In order to test the performance of the PCDM, we are addressing two deformation regimes: static behavior and dynamical response. We are then able to measure the response with light actuation, as the influence function matrix with respect to the number of projected virtual actuators; the response with frequency, including response time, resonance frequency, with respect to the different applied patterns, and with respect to the number of virtual actuators; finally, the response in open/close loop.
\end{abstract}

Keywords: Deformable mirror, Photo-controlled deformable mirror, Wavefront shaping, Adaptive Optics, Digital Micromirror Device, DMD.

\section{INTRODUCTION}

Several research groups around the world are currently involved in the design of highly performing adaptive optical (AO) systems as well as for next generation instrumentation of $10 \mathrm{~m}$-class telescopes than for future extremely large optical telescopes.

Wavefront correction like adaptive optics systems are based on a combination of three elements, the wavefront sensor for the measurement of the shape of the wavefront arriving in the telescope, the deformable mirror is the correcting element, and finally the real time computer closing the loop of the system at a frequency ranging from 0.5 to $3 \mathrm{kHz}$, in order to follow the evolution of the atmospherical perturbations (Fig. 1).

Four main types of AO systems have been built or are under development: Single-Conjugate Adaptive Optics (SCAO), Multi-Conjugate Adaptive Optics (MCAO), Multi-Object Adaptive Optics (MOAO), and Extreme Adaptive Optics (ExAO). These AO systems are associated with different types of WaveFront Sensors (WFS), combined with natural guide stars or laser guide stars, and different architectures of Deformable Mirrors (DM). Numerous science cases will use these AO systems, SCAO, the "classical" AO system will provide accurate narrow field imagery and spectroscopy, $\mathrm{MCAO}$, wide field imagery and spectroscopy, MOAO, distributed partial correction AO, and high dynamic range AO for the detection and the study of circumstellar disks and extra-solar planets. Corrected fields will vary from few arcsec to several arcmin. 


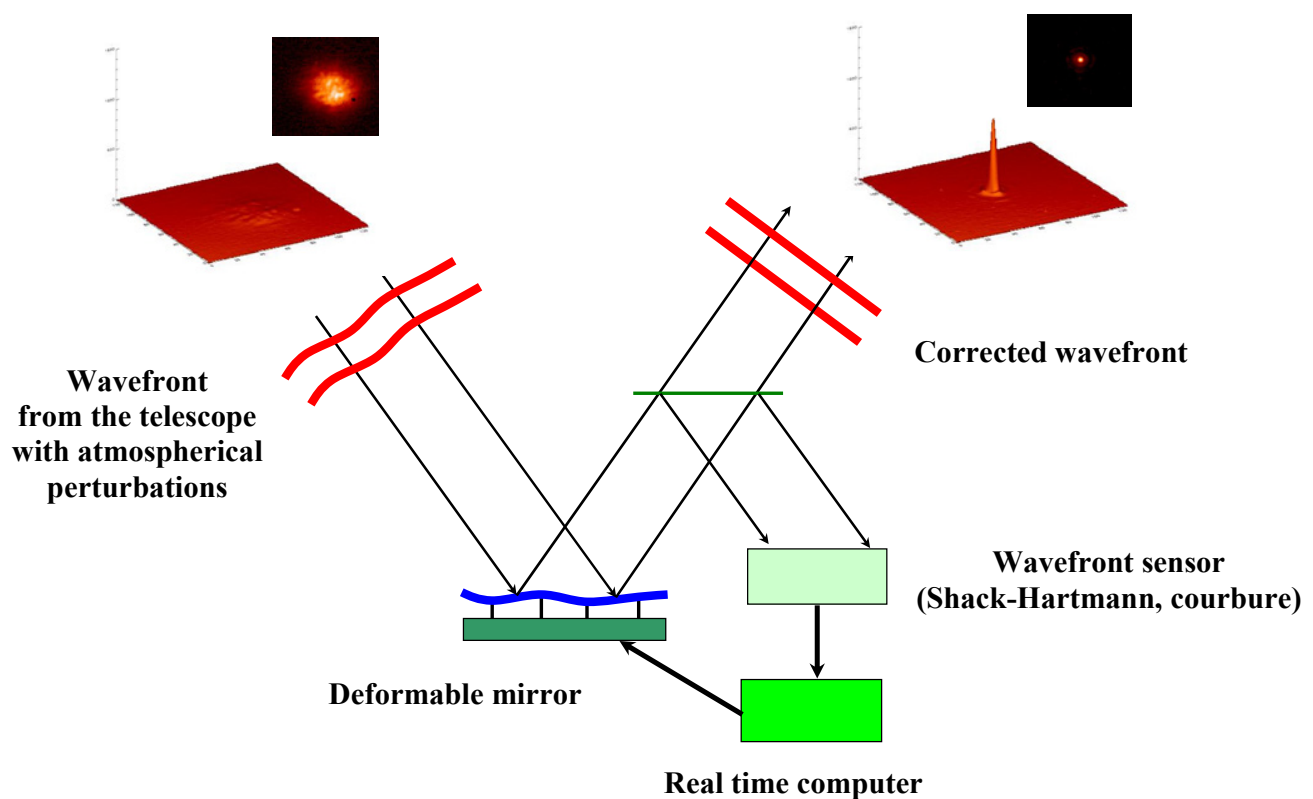

Fig. 1: Schematic of a wavefront correction system

These systems require a large variety of deformable mirrors with very challenging parameters. For a $8 \mathrm{~m}$ telescope, the number of actuators varies from a few 10 up to 5000; these numbers increase impressively for a $40 \mathrm{~m}$ telescope, ranging from a few 100 to over 50000 , the inter-actuator spacing from less than $200 \mu \mathrm{m}$ to $1 \mathrm{~mm}$, and the deformable mirror size from $10 \mathrm{~mm}$ to a few $100 \mathrm{~mm}$. Conventional technology cannot provide this wide range of deformable mirrors. The development of new technologies based on micro-opto-electro-mechanical systems (MOEMS) is promising for future deformable mirrors. The major advantages of the micro-deformable mirrors (MDM) are their compactness, scalability, and specific task customization using elementary building blocks. This technology permits the development of a complete generation of new mirrors. However this technology has also some limitation. For example, pupil diameter is an overall parameter and for a $40 \mathrm{~m}$ primary telescope, the internal pupil diameter cannot be reduced below $0.5 \mathrm{~m}$. According to the maximal size of the wafers (8 inches), a deformable mirror based on MOEMS technology cannot be build into one piece. New AO architectures have been proposed to avoid this limitation. ${ }^{1}$

LAM is involved since several years in conception of new MOEMS devices as well as in characterization of these components for the future instrumentation of ground-based and space telescopes. These studies include programmable slits for application in multi-object spectroscopy (JWST, European networks, EUCLID, BATMAN), deformable mirrors for adaptive optics, and programmable gratings for spectral tailoring.

We are particularly engaged in a European development of micromirror arrays (MMA) called MIRA for generating reflective slit masks in future Multi-object spectroscopy (MOS) instruments; this technique is a powerful tool for space and ground-based telescopes for the study of the formation and evolution of galaxies. MMA with $100 \times 200 \mu \mathrm{m}^{2}$ singlecrystal silicon micromirrors were successfully designed, fabricated and tested. Arrays are composed of 2048 micromirrors ( $32 \times 64)$ with a peak-to-valley deformation less than $10 \mathrm{~nm}$, a tilt angle of $24^{\circ}$ for an actuation voltage of $130 \mathrm{~V}$. The micromirrors were actuated successfully before, during and after cryogenic cooling, down to $162 \mathrm{~K}$. The micromirror surface deformation was measured at cryo and is below $30 \mathrm{~nm}$ peak-to-valley. ${ }^{2,3}$ In order to fill large focal planes (mosaicing of several chips), we are currently developing large micromirror arrays integrated with their electronics.

LAM is also leading the conception and realization of new MOEMS-based instruments. We are developing a 2048x1080 Digital-Micromirror-Device-based (DMD) MOS instrument to be mounted on the Telescopio Nazionale Galileo (TNG) and called BATMAN. A two-arm instrument has been designed for providing in parallel imaging and spectroscopic capabilities. BATMAN on sky is of prime importance for characterizing the actual performance of this new family of MOS instruments, as well as investigating new observational modes on astronomical objects, from faint 
and remote galaxies to active areas in nearby galaxies and small bodies of the solar system. This instrument will be placed at TNG by $2022 .{ }^{4}$

In this paper, we propose an innovative technology for a new type of membrane deformable mirror, made by coupling a reflective polymeric membrane with a monolithic non-pixelated photoconductive substrate.

\section{DEFORMABLE MIRRORS}

Three main Micro-Deformable Mirrors (MDM) architectures are under study in different laboratories and companies. First, the bulk micro-machined continuous-membrane deformable mirror, studied by Delft University and OKO company, is a combination of bulk silicon micromachining with standard electronics technology $y^{5}$ This mirror is formed by a thin flexible conducting membrane, coated with a reflective material, and stretched over an electrostatic electrode structure. This mirror shows a very good mirror quality, but the mean deformed surface is a concave surface, and the number of actuators cannot be scalable to hundreds of electrodes. Second, the segmented, micro-electro-mechanical deformable mirror realized by Iris $\mathrm{AO}^{6}$ consists of a set of segmented piston-tip-tilt moving surfaces, fabricated in dense array. For adaptive optics application, the wavefront has to be properly sampled, increasing the number of actuators for a given number of modes to be corrected. Third, the surface micro-machined continuous-membrane deformable mirror made by Boston Micromachines Corporation (BMC) is based on a single compliant optical membrane supported by multiple attachments to an underlying array of surface-normal electrostatic actuators ${ }^{7}$. The efficiency of this device has been demonstrated recently in several AO system, including the GPI instrument on Gemini telescope. The third concept is certainly the most promising architecture, but it shows limited strokes for large driving voltages, and mirror surface quality may need further improvement for Extreme AO. All these devices are based on silicon or polysilicon materials.

\subsection{BMC Deformable Mirror}

BMC produces the most advanced MEMS deformable mirrors. The concept is based on an array of electrostatic actuators linked one by one to a continuous top mirror (Fig. 2). Their main parameters are approaching the requirements values, i.e. large number of actuators (up to 4096, see Fig. 2), large stroke (up to $5.5 \mu \mathrm{m}$ ), good surface quality, but they still need large voltages for their actuation (150-250V).

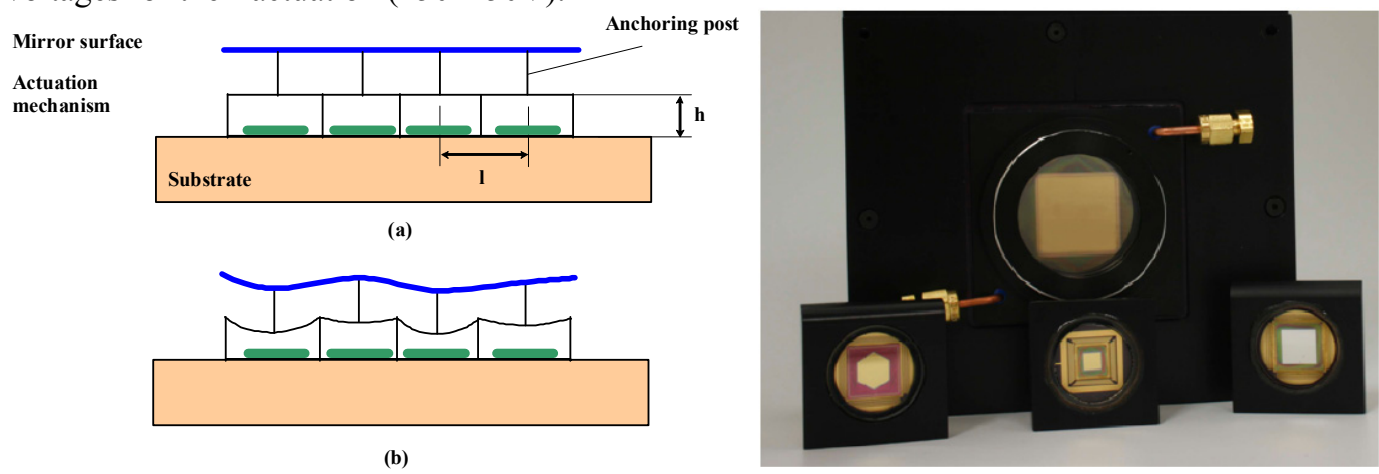

Fig. 2: Continuous membrane MDM from BMC

\subsection{Iris AO Deformable Mirror}

IRIS AO is producing segmented piston tip tilt mirrors with very flat mirrors. An exploded-view schematic diagram is presented in Fig. 3a. The DM array is paved by 37 hexagonal segments with a size of $700 \mu \mathrm{m}$ from vertex-to-vertex, with a $606 \mu \mathrm{m}$ pitch. The segment is capable of moving in piston/tip/tilt motions (PTT). In Fig. 3a, scaling is highly exaggerated in the vertical direction. In Fig. $3 \mathrm{~b}$ is shown a die photograph of a 111-actuator 37-piston/tip/tilt-segment DM with $3.5 \mathrm{~mm}$ inscribed aperture (PTT111 device). The DM is manufactured using typical MEMS and integrated circuit materials such as polycrystalline silicon (polysilicon), silicon dioxides, silicon nitrides, and a proprietary bimorph material with similar coefficient of thermal expansion (CTE) to that of polysilicon. The s-shape of the bimorph flexures that elevates the DM segment is a result of engineered residual tensile stresses in the bimorph and actuator-platform polysilicon. After the DM is fabricated using highly stable MEMS materials, it is mounted onto a ceramic pin-grid array (PGA) package using an epoxy. The DM is sealed in nitrogen by epoxying a cover window over the DM. 


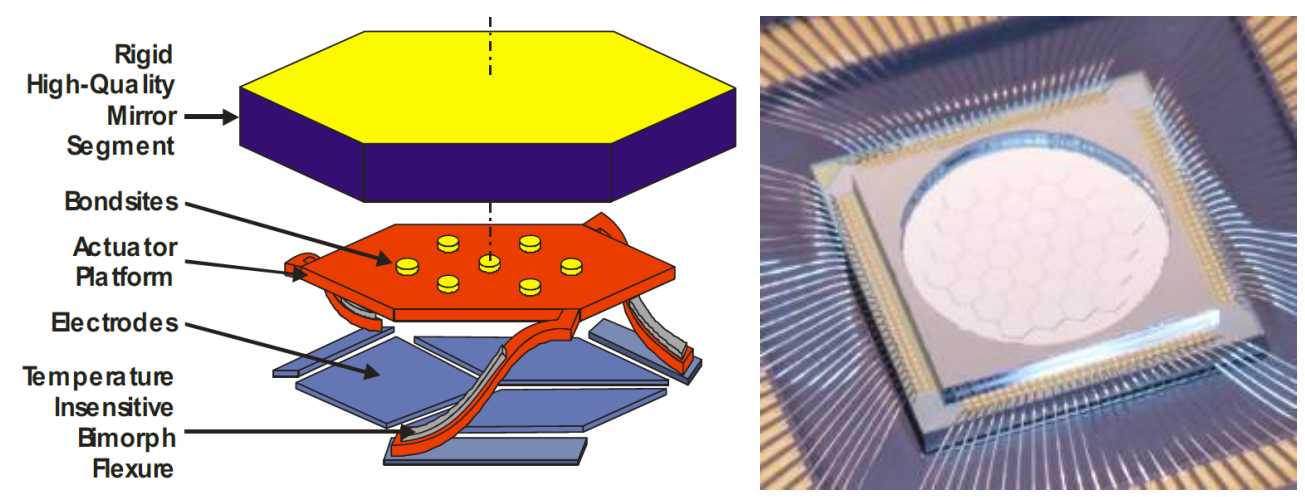

Fig. 3: Segmented MDM from Iris AO (a) Concept of one segment of the mirror;

(b) Die photograph of a 111-actuator 37-piston/tip/tilt-segment DM with $3.5 \mathrm{~mm}$ inscribed aperture (PTT111 device)

To actuate the DM, the red actuator-platform layer is held at ground potential and the three diamond-shaped electrodes are energized at different electrical potentials. Applying the same voltage to all three electrodes pulls the segment in a piston motion toward the electrodes. A differential voltage across the electrodes results in tip and tilt motions. Because the positioning is highly repeatable, the DM segment motion can be calibrated, thus linearizing the DM position into orthogonal coordinates.

We tested the PTT $111 \mathrm{DM}$ from Iris AO. The device could be operated successfully from ambient to $160 \mathrm{~K}$. An additional, mainly focus-like, $500 \mathrm{~nm}$ deformation of the entire mirror is measured at $160 \mathrm{~K}$; we were able to recover the best flat in cryo by correcting the focus and local tip-tilts on all segments, reaching $12 \mathrm{~nm}$ rms. Finally, the goal of these studies is to test DMs in cryo and vacuum conditions as well as to improve their architecture for stable operation in harsh environments. ${ }^{8}$

\section{Photo Controlled Deformable Mirror (PCDM)}

\subsection{Photo Controlled Deformable Mirror (PCDM) concept}

We propose an innovative technology for a new type of membrane deformable mirror, made by coupling a reflective polymeric membrane with a monolithic non-pixelated photoconductive substrate. The device is called continuous photocontrolled deformable mirror (PCDM). The general scheme of the PCDM is shown in Fig. 4.

A light flux with given shape and intensity distribution, on the back side of the photoconductor, opposite to the reflective side. By applying a voltage to the system, an electrostatic pressure, exerted by the photo-generated charge carriers, appears and deforms the thin membrane $(\Delta \mathrm{M})$ according to the Poisson equation. ${ }^{9,10,11}$

Unlike the other actuation mechanisms, this allows to obtain a continuous actuation field, without the typical segmented actuators pattern. Furthermore, it leads to a strong simplification to the driving electronics, for example by eliminating hundreds of cables used for the deformation control.

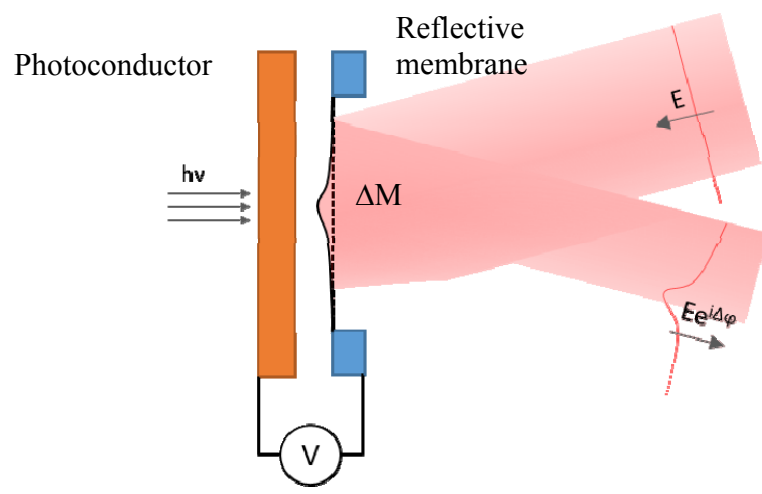

Fig. 4: Scheme of a PCDM showing the photoconductor, the reflective membrane and the local deformation due to the illumination. 
This innovative technology allows for the development of deformable mirrors that are almost free of boundary regarding the size, shape and number of actuators since the light pattern projected on the backside of a photoconductor determines these parameters.

\subsection{PCDM model}

A model of the PCDM has been developed. In order to have high performance PCDMs, two parameters related to the features of the photoconductor are crucial, the dynamic range $\Delta \mathrm{M}$ and the response time $\tau$. The first parameter is related to the maximum displacement induced in the membrane by a uniform illumination, the second determines how fast can be the mirror response upon light stimulus.

Considering a thin membrane, the relative displacement of the mirror $\Delta \mathrm{M}$ due to a uniform illumination can be determined based on a simple electrical model as follows:

$$
\Delta M=\frac{\varepsilon \Phi^{2}}{32 T} \frac{V_{e f f}^{2}}{d^{2}}\left[1-\frac{1}{\left(1+\widetilde{d_{p c}} / d\right)^{2}}\right]
$$

where $T$ is the membrane tension, $\Phi$ the membrane diameter, $V_{e f f}$ is the applied voltage, $\widetilde{d_{p c}}=d_{p c} / \varepsilon_{r}$ is the effective photoconductor thickness (being $\varepsilon_{\mathrm{r}}$ its relative permittivity), and $d$ is the distance between the semiconductor and the membrane. We notice that $\Delta M$ increases when the effective photoconductor thickness $\widetilde{d_{p c}}$ is large with respect to $d$.

As for the response time $\tau$, we can write:

$$
\tau=\tau_{r e l}\left(1+\frac{\widetilde{d_{p c}}}{d}\right)
$$

where $\tau_{\text {rel }}=\varepsilon_{0} \varepsilon_{\mathrm{r}} / \sigma_{\mathrm{pc}}$, which is the photoconductor dielectric relaxation time (being $\sigma_{p \mathrm{c}}$ its photoconductivity) and clearly dominates the response time. In addition, it can be seen that maximizing $\widetilde{d_{p c}}$ and hence $\Delta M$, comes at the expense of $\tau$ : once that $\widetilde{d_{p c}}$ is made sufficiently larger than $d$, there is no point in further increasing it, because the gain in $\Delta M$ is small, whereas $\tau$ is negatively affected.

To summarize, in order to develop an efficient PCDM, a suitable photoconductor must show:

i) a low dielectric constant;

ii) a large thickness (i.e. of the order of at least some microns);

iii) a sufficient charge carrier mobility.

These important guidelines are related to the photoconductor and the optoelectronic part; as for the mechanical deformation, it is necessary to solve the mechanical problem and this has been done by means of finite elements analysis having as input the photo-generated electrostatic pressure.

We report here an example of the response of a PCDM with a $20 \mathrm{~mm}$ diameter when illuminated with a defined light pattern and the corresponding deformation calculated by finite element analysis with ANSYS® (Fig. 5). The illumination pattern turns into the electrostatic pressure pattern.
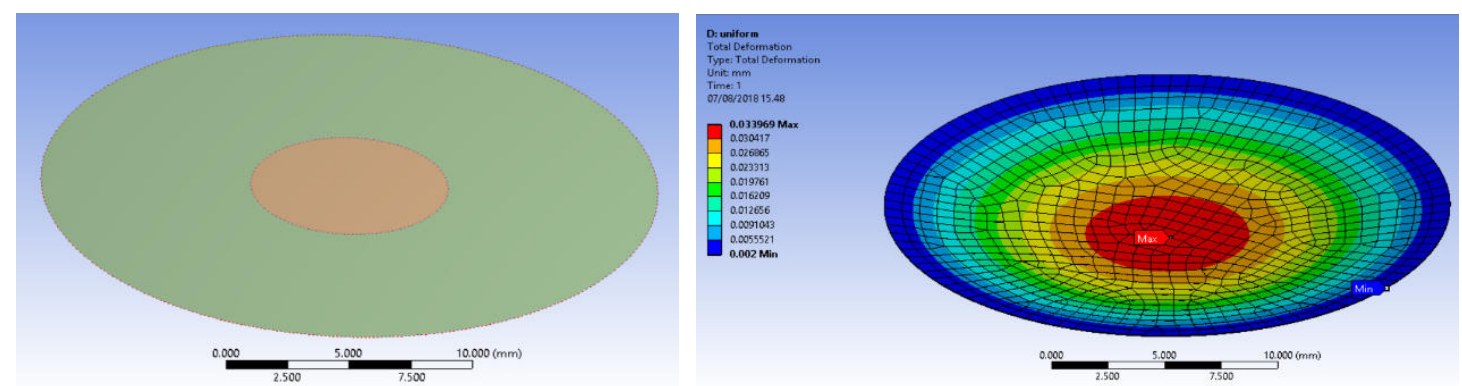

Fig. 5: On the left: geometry of the illumination pattern; on the right: the corresponding deformation of the membrane. 
Such approach is powerful, since by the optoelectronic model it is possible to define the pressure and solve both the static and dynamic problems considering also the effect of the surrounding medium (air).

\section{PCDM DEVICES}

Several types of PCDMs have been designed, modeled, fabricated and tested. They are based on different photoconductors: Zinc Selenide, organic substrate, Silicon, Cadmium Sulfide. The membrane is always polymeric with a reflective coating.

\subsection{Zinc Selenide photoconductor}

A PCDM with a 2-inch-diameter clear aperture based on zinc selenide $(\mathrm{ZnSe})$ has been designed and realized. This semiconductor has a relatively high charge carrier mobility (540 and $30 \mathrm{~cm}^{2} / \mathrm{s}$ for electrons and holes, respectively, in a single crystal), relatively low dielectric constant (9.25), and availability in the form of large substrates. A 2-inch aperture PCDM was assembled using a 2-inch diameter, 2-mm-thick polycrystalline ZnSe substrate with a transparent ITO electrode $(40 \Omega / \mathrm{sq})$ on one side and a $5 \mu \mathrm{m}$ thick aluminized nitrocellulose membrane with a planarity within two waves per inch peak to valley placed at $75 \mu \mathrm{m}$ from the photoconductor by means of suitable spacers. ${ }^{11}$

The PCDM was then characterized using an optical setup equipped with a 670-nm laser source and a Shack-Hartmann wavefront sensor (WFS) to measure the membrane shape. The membrane tension was calculated measuring its maximum displacement at the center as a function of the applied electric field in dark conditions and fitting the data adopting the approximation of thin membranes. The result was $68 \mathrm{~N} / \mathrm{m}$.

The membrane displacement at the center was also measured as a function of light intensity at different frequencies in conditions of uniform illumination. The largest measured displacement $(16 \mu \mathrm{m})$ was actually limited by the dynamic range of the wavefront sensor. The temporal response was characterized by illuminating the PCDM locally on an area of $1 \mathrm{~cm}^{2}$, simulating the action of a single conventional actuator and measuring the membrane shape variation when the light stimulus is applied (raise time) and when light is removed (fall time). Very slow response times (rise and fall times $10 \%-90 \%>300 \mathrm{~ms}$ ) were measured for the PCDM in air due to viscous damping.

Adaptive optics tests were performed to determine PCDM capabilities. The PCDM was driven by a DMD (digital micromirror device) light projector equipped with a blue LED (peaked at $450 \mathrm{~nm}$ ) using a light pattern with 32 discrete light actuators covering a diameter of $45 \mathrm{~mm}$. Maximum light intensity was $13 \mathrm{~mW} / \mathrm{cm}^{2}$, and working conditions of $400 \mathrm{Vpp}$ and $500 \mathrm{~Hz}$ were chosen to optimize the PCDM stroke while minimizing the impact of the AC power supply oscillations on the membrane position. To demonstrate the capabilities of the PCDM to generate complex shapes, Zernike polynomials were generated up to Zernike number 14 (tetrafoil Z4;-4), except for numbers 0,1 , and 2 (piston, tip and tilt) as shown in Fig. 6. Even in this case, the wavefront error was low (within $\lambda / 10$ ) and the Zernike mode amplitude was decreasing for increasing order, as expected due to the low pass behavior of the membrane when assuming shapes with a high number of features.

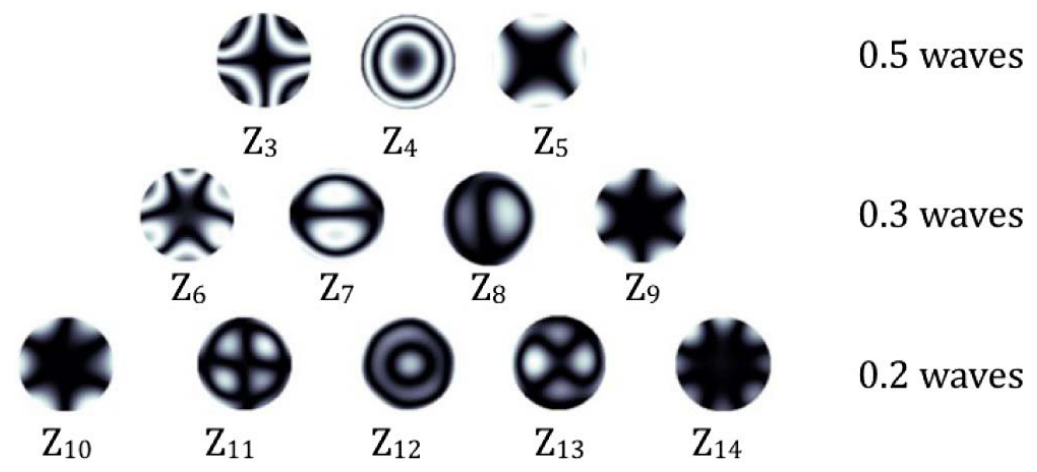

Fig. 6: Interferograms showing the membrane shape when using the PCDM to generate the first 14 Zernike polynomials (except for numbers 0,1 , and 2). From reference 11. 


\subsection{Organic photoconductor}

A prototype of PCDM based on an organic photoconductive layer has been manufactured and tested as optical valve. ${ }^{12}$

The key element of such PCDM is the photoconductive layer consisting in a mixture of P3HT/PCBM with an arylamine (m-MTDATA) dispersed in a polystyrene matrix (see Fig. 7 for the chemical structure of the different components).
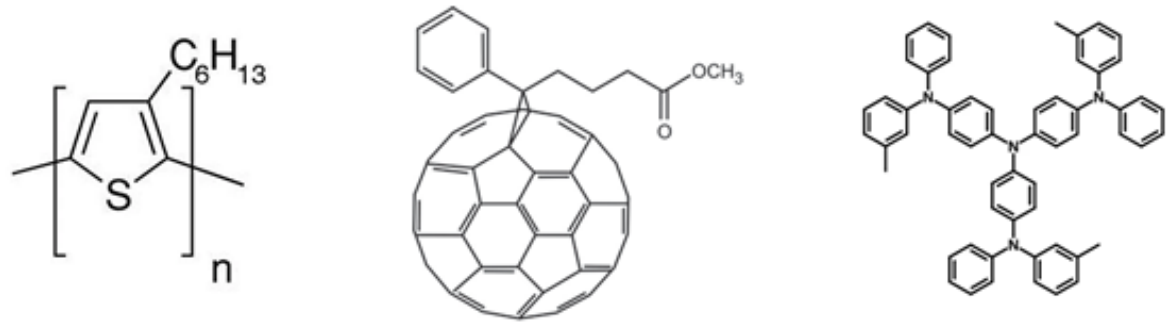

Fig. 7: Chemical structure of the main components of the photoconductor layer developed for the organic PCDM, from left to right: P3HT, PCDM and m-MTDATA.

The P3HT/PCBM is a well-known organic system exploited in organic solar cells, photodetectors and it is characterized by a good photogeneration yield and a strong absorption in the visible. The m-MTDATA is an aryl amine widely exploited as positive charge transport material and this is the role in the film. PS has been chosen as matrix thanks to its low content of charge traps and the compatibility with the other components.

According to the photoconductor guidelines, it was necessary to obtain a thick film, which was not common with such organic system. By balancing the amount of the different components, it was possible to have a 40 um thick film on a glass substrate coated with ITO as electrode. The PCDM assembly is reported in Fig. 8, together with the characterization in terms of maximum displacement when illuminated with uniform light at $525 \mathrm{~nm}$.

We notice how the displacement increases with the light intensity since more charges are photogenerated and a larger electrostatic pressure created. Moreover, the displacement increases with the applied voltage as expected. The magnitude of the displacement is big enough to induce a remarkable change in the wavefront reflected by the mirror.
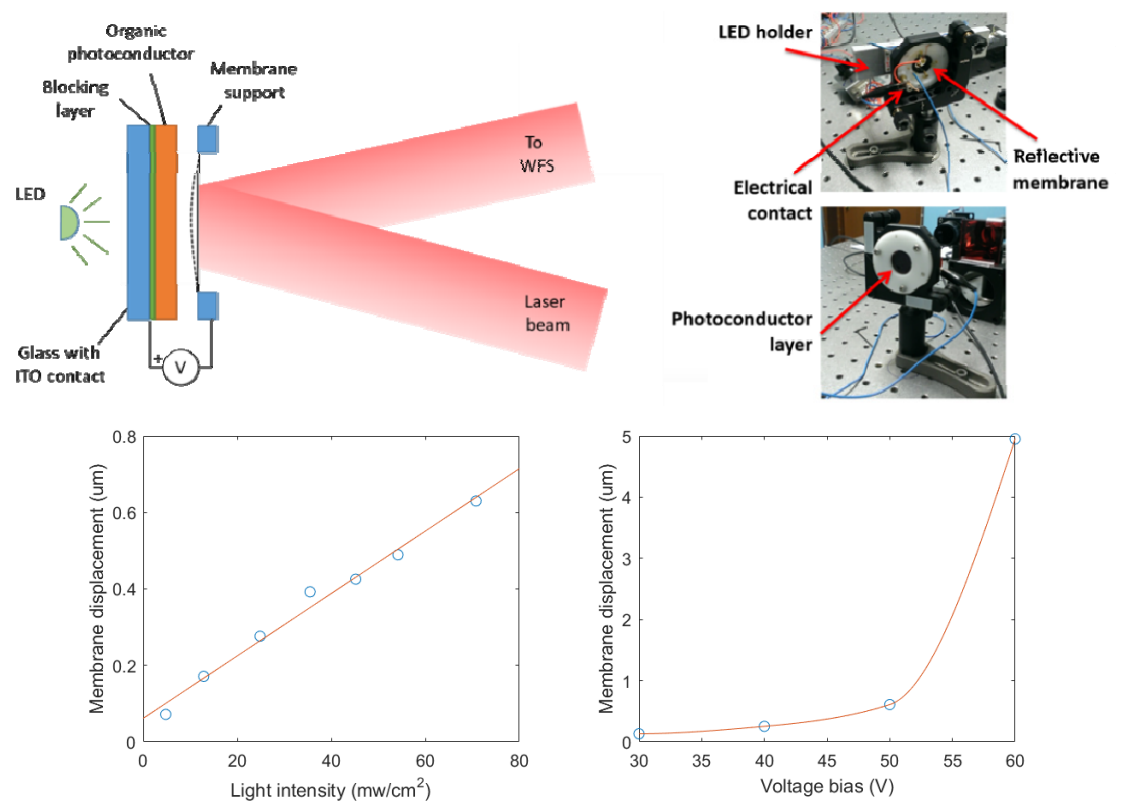

Fig. 8: On top, the scheme of the PCDM based on the organic photoconductor.

On the bottom, the membrane displacement as function of the light intensity (at $525 \mathrm{~nm}$ ) and on the applied voltage. 
Based on this device, a set-up where it works as a light valve was built (Fig. 9) and tested. When the light is OFF, a wellfocused spot is found on the camera; by switching on the illumination, a $80 \%$ decrease of the light intensity on the focal area occurs, since the light is spread out over a much larger area. This confirms the potential use of the organic PCDM as a fully light driven valve.

(a)

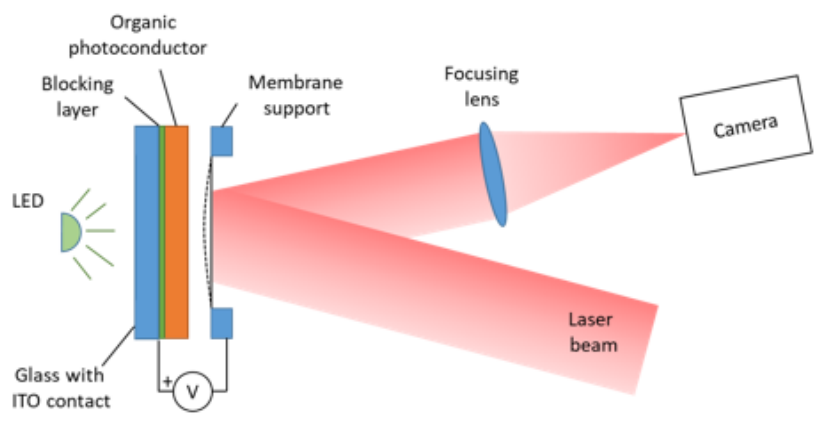

(b)

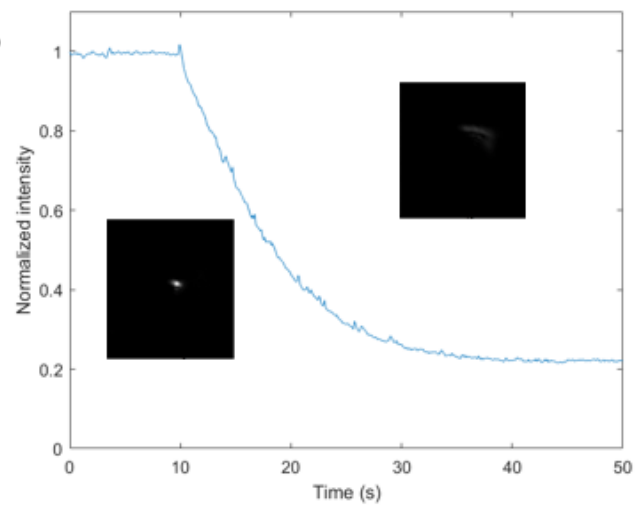

Fig. 9: a) Scheme of the set-up used to demonstrate the use of the organic PCDM as a light valve; b) light intensity variation on the CMOS camera. Inset: image of the focal point before and after illumination. The working conditions were the following: DC bias $60 \mathrm{~V}$, illumination intensity of $70.8 \mathrm{~mW} / \mathrm{cm}^{2}$ at $525 \mathrm{~nm}$.

\subsection{Silicon / Cadmium Sulfide photoconductors}

Considering different photoconduction performance, we have developed a PCDM based on a silicon and Cadmium Sulfide (CdS) photoconductor. ${ }^{13}$

The main differences in between the two are the charge mobility and the intrinsic resistivity. Silicon is better in terms of mobility, the $\mathrm{CdS}$ is better for the internal resistance.

The parameters of the considered device are the following:

- The photoconductor is a silicon wafer with an ITO coating on one side;

- The membrane is a polymeric membrane with coatings on both sides;

- The insulating mechanics is made of ABS and is 3D printed;

- The Aluminium mechanics gives the stiffness to the device.

Table 1 shows the parameters considered in this development. The polymeric membrane must be conductive thanks to the reflective aluminium coating on top.

\begin{tabular}{|l|l|}
\hline \multicolumn{1}{|c|}{ Electro-mechanical parameters of the system } & \multicolumn{1}{|c|}{ Value or range } \\
\hline Thickness of the membrane & $5 \mu \mathrm{m}$ \\
\hline Thickness of the silicon slab & $3 \mathrm{~mm}$ \\
\hline Capacity of the silicon & $13 \mathrm{pF}$ \\
\hline Distance between the membrane and silicon $\mathrm{d}_{\mathrm{I}}$ & $100-150 \mu \mathrm{m}$ \\
\hline Resistance of the silicon $\mathrm{R}_{\mathrm{pc}}$ & $60 \mathrm{k} \Omega$ \\
\hline Diameter of the silicon & $25.4-50.8 \mathrm{~mm}$ \\
\hline Diameter of the membrane & $25.4-50.8 \mathrm{~mm}$ \\
\hline Power supply & $100-180 \mathrm{~V}(\mathrm{DC})$ \\
\hline
\end{tabular}

Table 1: Electro-mechanical data of the Silicon-based PCDM.

Two dynamical regimes must be considered: the power supply frequency in AC mode in order to permit to the silicon photoconductor to recombine the charges, and the system operating frequency, i.e. the light pattern reconfiguration frequency (Fig. 10). The power supply frequency must be higher than the operating frequency (usually $\mathrm{kHz}$ range for atmospheric turbulence compensation) as well as the membrane resonance frequency, in order to minimize the ripples of the membrane and have only the deformation induced by the light pattern. 


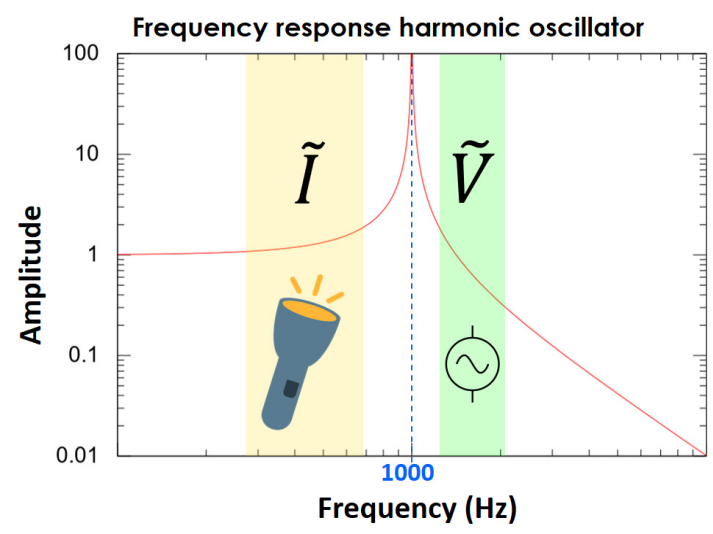

Fig. 10: Frequency regimes for the operating system (light pattern modulation) and the power supply frequency

From the developed model of the device as well as some experimental data (light power, charges generation, membrane tension), the foreseen deformation of the membrane with respect to the operating voltage is shown in Fig. 11 in the case of uniform illumination. The $3 \mathrm{~mm}$-thick photoconductor is Silicon $\left(\varepsilon_{\mathrm{Si}}=11.7\right)$, the air-gap $\left(\varepsilon_{\text {air }}=1\right)$ is $125 \mu \mathrm{m}$, and the polymeric membrane has a diameter of $50.8 \mathrm{~mm}(2$ ") and a mechanical tension of $50 \mathrm{~N} / \mathrm{m}$.

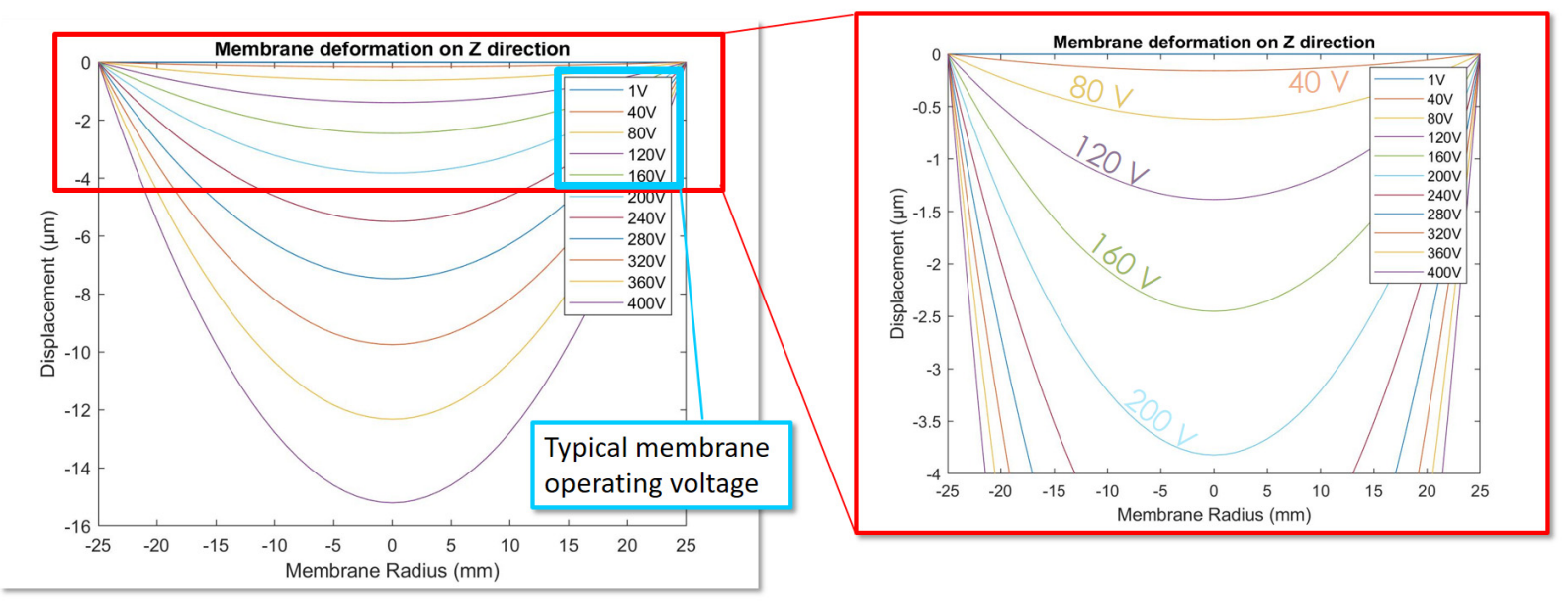

Fig. 11: Membrane deformation with respect to the operating voltage.

The $3 \mathrm{~mm}$-thick photoconductor is Silicon $\left(\varepsilon_{\mathrm{Si}}=11.7\right)$, the air-gap $\left(\varepsilon_{\text {air }}=1\right)$ is $125 \mu \mathrm{m}$, and the polymeric membrane has a diameter of $50.8 \mathrm{~mm}(2 ")$ and a mechanical tension of $50 \mathrm{~N} / \mathrm{m}$.

The mechanical design of the assembled device has been published elsewhere. ${ }^{13}$ The same scheme can be used with $\mathrm{CdS}$ photoconductor.

\section{PCDM CHARACTERIZATION SET-UPS}

In order to test the performance of the PCDM, we are addressing two deformation regimes: static behavior and dynamical response. We are then able to measure the response with light actuation, as the influence function matrix with respect to the number of projected virtual actuators; the response with frequency, including response time, resonance frequency, with respect to the different applied patterns, and with respect to the number of virtual actuators; finally, the response in open/close loop.

The Laboratoire d'Astrophysique de Marseille has developed over the last few years an expertise in the characterization of micro-optical components. Our expertise in small-scale deformation characterization on the surface of micro-optical components has been conducted initially within the framework of the NASA study of a multi-object infrared spectrograph equipped with MOEMS-based slit masks for the JWST. 
Two set-ups will be used for the complete characterization of the PCDM:

- $\quad$ an interferometric bench for static and high spatial resolution characterization of the PCDM surface; we will address the full PCDM surface, using uniform sources or fixed patterns, with different source flux

- a functional bench for low frequency and high frequency characterization; the pattern generator is a DMD with more than 2 millions micromirrors, and through a dynamical excitation, the deformation modes generated on the PCDM surface will be either measured by a Shack Hartmann sensor (full surface characterization) or a fast photodiode (dynamical response, resonances)

\subsection{Interferometric bench}

An interferometric characterization bench has been developed in order to measure the shape and the deformation parameters of these devices. All optical characterizations in static or dynamic behavior are performed, including measurements of optical surface quality at different scales, actuators stroke, maximum mirror deformation and cut-off frequency. This bench is a high-resolution and low-coherence Twyman-Green interferometer (Fig. 12) The light source is an halogen lamp with an interference filter (typical example: $\lambda_{0}=650 \mathrm{~nm}, \Delta \lambda=10 \mathrm{~nm}$ ). Monitoring the temporal light coherence, this illumination avoids all extraneous fringes induced by classical high coherence sources such as lasers. Conceived as a modular bench, a simple lens change offers two magnification configurations: (1) high in-plane resolution or (2) large field of view authorizing either a very sharply (around $4 \mu \mathrm{m}$ ) analysis of the micro-mirror structure inside a small field (typically $1 \mathrm{~mm}$ ), or the whole device study with larger size (up to $40 \mathrm{~mm}$ ). For the high-resolution configuration, diffraction limit $(\mathrm{N}=3)$ is reached. Out-of-plane measurements are performed with phase-shifting interferometry showing very high resolution (standard deviation<1nm). A picture of the bench is shown in Fig. 12, mounted on a damped optical table, and surrounded by a Plexiglas enclosure. Features such as optical quality or electromechanical behavior are extracted from these high precision three-dimensional component maps. Range is increased without loosing accuracy by using two-wavelength phase-shifting interferometry authorizing large steps measurements. ${ }^{14}$

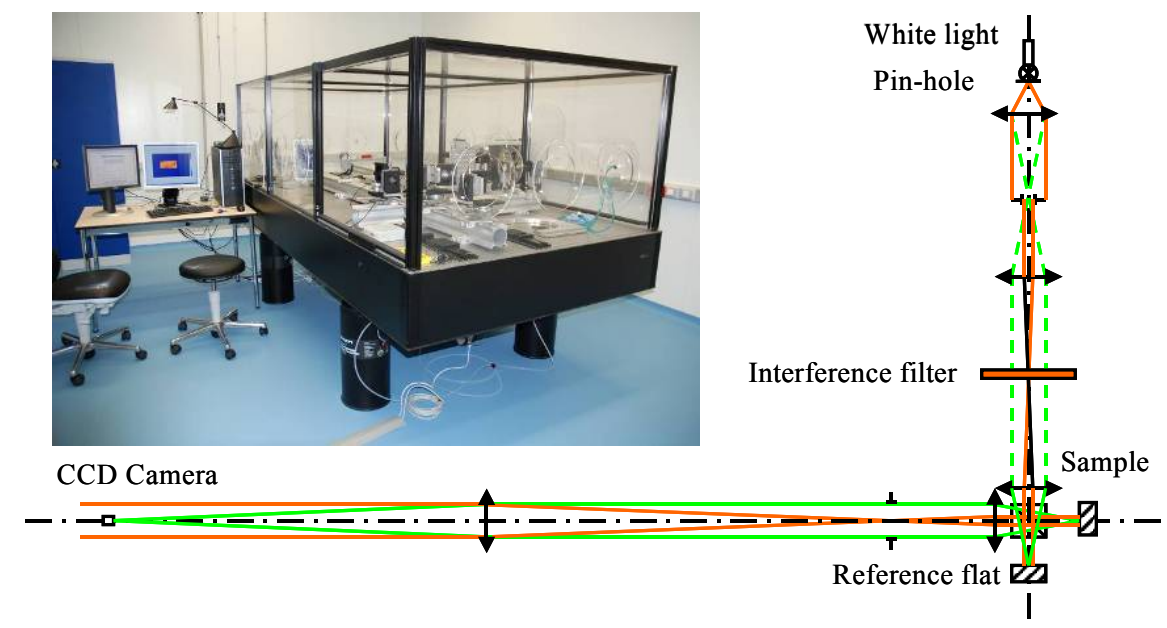

Fig. 12: Schematic description / picture of our interferometric measurement bench.

\subsection{Functional bench}

The functional bench is based on an original set-up used at LAM for recording Computer Generated Holograms (CGH) on photosensitive plates. Thanks to a DMD as a mask generator, we have been able to develop a new type of Fouriercoded gray scale holograms. ${ }^{15}$

Figure 13 shows our functional bench. The DMD, controlled by the formatter board ${ }^{16}$ is illuminated by a collimated beam from a white source, and redirects the light toward the back of the PCDM photoconductor. The beam is illuminating the entire DMD and the light power is homogeneous on the photoconductor. The pattern reproduced by the DMD has to be projected onto the photoconductor of the PCDM as precisely as possible, so the plate is illuminated through an Offner relay with a magnification of $1: 1$. This relay provides a nearly aberration free beam and has the advantage of being compact. The actuation of the PCDM is then controlled by a light pattern modulated by the DMD chip, generating a reprogrammable actuator pattern projected on the photoconductor. Finally, a post-PCDM imaging 
system "looks" at the PCDM membrane surface. The imaging system consists of two lenses, with two different sensors: a Shack Hartmann sensor for full surface characterization or a fast photodiode for the dynamical response and resonances measurement. This system is an afocal assembly for PCDM imaging in situ and in real time. Magnification is tuned by changing properly the pair of lenses, from a value of 1 up to 4 .

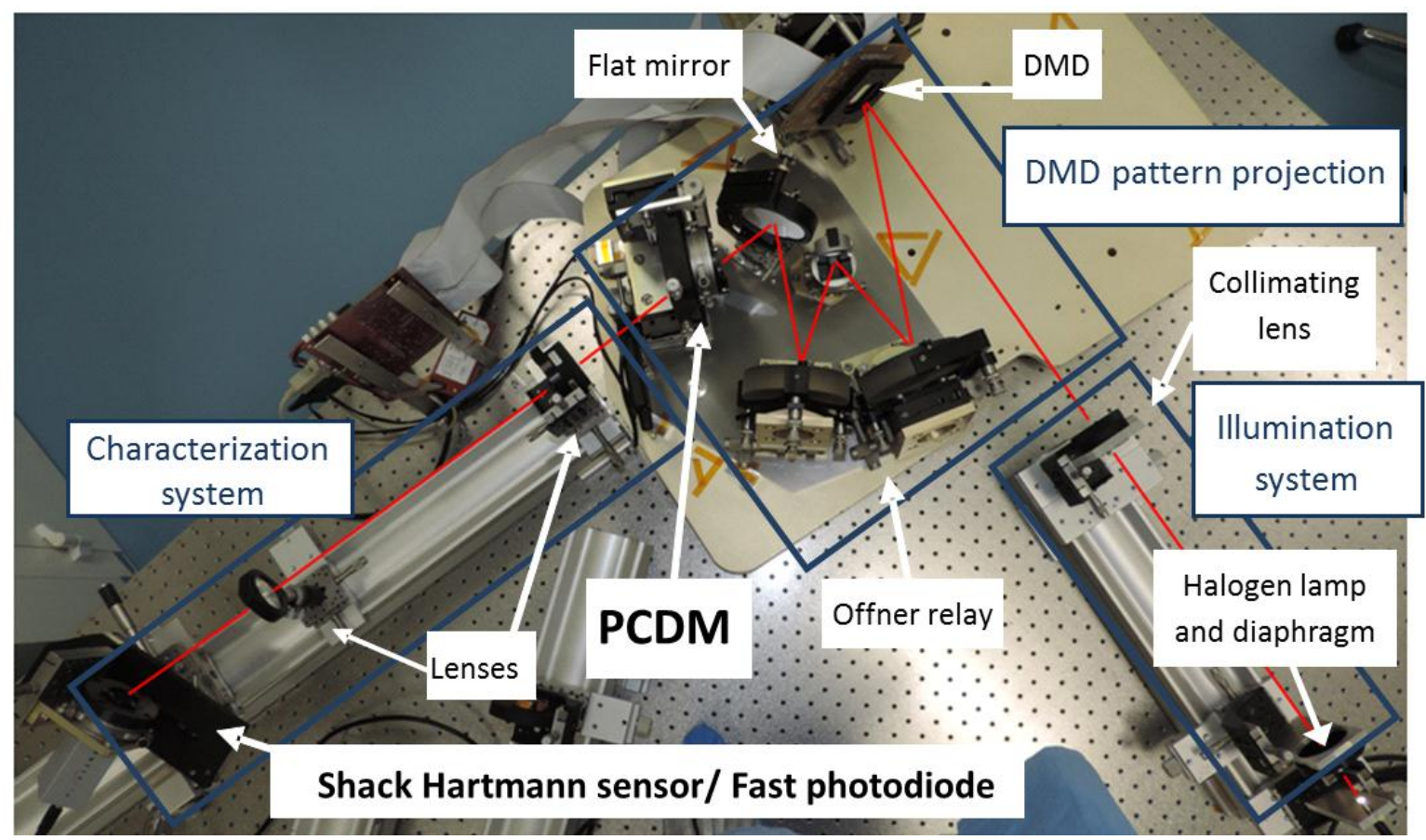

Fig. 13: Picture of the functional bench dedicated to PCDM characterization; it is based on an illumination unit towards the DMD, an imaging optical system based on a 1:1 magnification Offner relay from the DMD plane to the PCDM plane for the photoconductor illumination, and a post-PCDM imaging system for membrane surface characterization.

\section{Pattern generator}

Digital Micromirror Devices (DMD) from Texas Instruments could act as reconfigurable mask generator. The largest DMD chip developed by TI features 2048 x 1080 mirrors on a $13.68 \mu \mathrm{m}$ pitch, where each mirror can be independently switched between an ON $\left(+12^{\circ}\right)$ position and an OFF $\left(-12^{\circ}\right)$ position (Fig. 14). This component has been extensively studied in the framework of an ESA technical assessment of using this DMD component ( $2048 \times 1080$ mirrors) for space applications (for example in EUCLID mission). Specialized driving electronics and a cold temperature test set-up have been developed. Our tests reveal that the DMD remains fully operational at $-40^{\circ} \mathrm{C}$ and in vacuum. A 1038 hours life test in space survey conditions $\left(-40^{\circ} \mathrm{C}\right.$ and vacuum) has been successfully completed. Total Ionizing Dose (TID) radiation tests, thermal cycling (over 500 cycles between room temperature and cold temperature, on a non-operating device) and vibration and shock tests have also been done; no degradation is observed from the optical measurements. These results do not reveal any concerns regarding the ability of the DMD to meet environmental space requirements. ${ }^{16}$

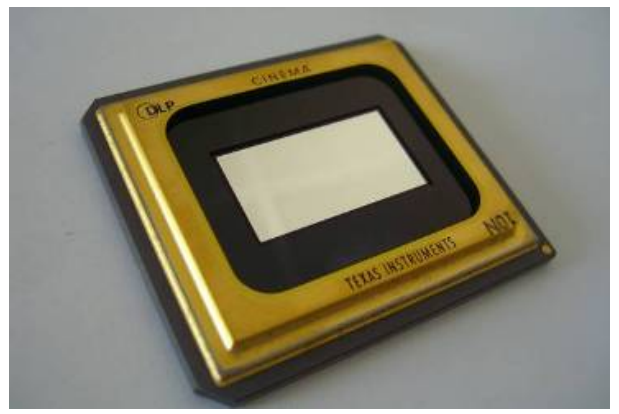

Fig. 14: DMD chip from Texas Instruments (2048 x 1080 micromirrors). 


\subsection{PCDM characterization plan}

The two-millions mirrors DMD will then be able to generate any number of actuators between 1 single actuator up to 2 million virtual actuators, to be projected on the PCDM. Software for generating the different patterns to be used in our characterization set-up, both in static and dynamic regimes will be developed.

In order to test the performance of the PCDM, we will address the following parameters:

- $\quad$ Response with light actuation: we will build the influence function matrix with respect to the number of projected virtual actuators, and measure the influence function overlap between actuators. We will also measure the efficiency of conversion of light intensity to surface deformation.

Response with frequency: response time when a light pattern is set (up and down time), resonance frequency, with respect to the different applied patterns, and with respect to the number of virtual actuators

Response at best flat: procedure and performance to be able to flatten the mirror surface, as operation starting point

Response with optical shapes (Zernike), overall stroke, actuator to actuator stroke, departure to the set Zernike mode

Response in open/close loop: need (or no need) to use a wave front sensor for reaching the mirror deformation shapes for a given accuracy / for a given refresh rate

Stability, reproducibility, aging: multiple measurements in time at short, medium and long scale

For controlling / testing the PCDM, we are developing the software command-control tools for generating all defined patterns to proceed to the complete characterization of the device.

\section{CONCLUSION}

Wavefront shaping systems in the field of Adaptive Optics for Astronomy, wide-field imaging in Microscopy and live cells/tissues imaging in Biology need efficient and reconfigurable Deformable Mirrors (DM).

The photo-controlled deformable mirror (PCDM) is a new type of membrane deformable mirror, made by coupling a reflective polymeric membrane with a monolithic non-pixelated photoconductive substrate. By sending a light flux with given shape and intensity distribution, thanks to a Digital Micromirror Device (DMD) on the back side of the photoconductor, opposite to the reflective side, we are able to tailor dynamically the projected virtual actuators in terms of number and shapes.

We have developed a device with a single crystalline semiconductor photoconductor wafer, either Silicon or Cadmium Sulfide. Modelling and opto-mechanical architecture have been completed and the devices are at integration level.

Interferometric and functional benches have been developed and will be used for the complete characterization of the DMs in two deformation regimes: static behavior and dynamical response; finally, the response in open/close loop will be tested.

\section{ACKNOWLEDGMENTS}

This work has been partly funded by the European Union H2020-OPTICON 3 program. 


\section{REFERENCES}

[1] F. Zamkotsian, K. Dohlen, "Prospects for MOEMS-based adaptive optical systems on extremely large telescopes", in Proceedings of the conference Beyond conventional Adaptive Optics, Venice, Italy (2001)

[2] S. Waldis, F. Zamkotsian, P.-A. Clerc, W. Noell, M. Zickar, N. De Rooij, "Arrays of high tilt-angle micromirrors for multiobject spectroscopy, " IEEE Journal of Selected Topics in Quantum Electronics 13, pp. 168-176 (2007).

[3] M. Canonica, F. Zamkotsian, P. Lanzoni, W. Noell, N. de Rooij, "The two-dimensional array of 2048 tilting micromirrors for astronomical spectroscopy," Journal of Micromechanics and Microengineering, 23 055009, (2013)

[4] Frederic Zamkotsian, Patrick Lanzoni, Nicolas Tchoubaklian, Harald Ramarijaona, Manuele Moschetti, Marco Riva, Marc Jaquet, Paolo Spano, William Bon, Mathieu Vachey, Luciano Nicastro, Emilio Molinari, Rosario Cosentino, Adriano Ghedina, Manuel Gonzalez, Walter Boschin, Paolo Di Marcantonio, Igor Coretti, Roberto Cirami, Filippo Zerbi, Luca Valenziano, " BATMAN @ TNG: Instrument integration and performance", SPIE conference on Astronomical Instrumentation 2018, Proc. SPIE 10702, Austin, USA, (2018)

[5] G. Vdovin, S. Middelhoek and P. M. Sarro, "Technology and applications of micromachined silicon adaptive mirrors", Opt. Eng., 36 (5), 1382-1390 (1997)

[6] M.A. Helmbrecht, M. He, C.J. Kempf, F. Marchis "Long-term stability and temperature variability of Iris AO segmented MEMS deformable mirrors", Proc. SPIE 9909, (2016)

[7] S. Cornelissen, T. G. Bifano "Advances in MEMS deformable mirror development for astronomical adaptive optics", in Proceedings of the SPIE conference on MOEMS 2012, Proc. SPIE 8253, San Francisco, USA (2012)

[8] Frederic Zamkotsian, Patrick Lanzoni, Rudy Barette, Michael Helmbrecht, Franck Marchis, Alex Teichman, "Operation of a MOEMS deformable mirror in cryo: challenges and results ", Micromachines, 8, 233; (2017)

[9] U. Bortolozzo, S. Bonora, J. P. Huignard, and S. Residori, "Continuous photocontrolled deformable membranemirror," Appl. Phys. Lett. 96, 251108 (2010)

[10] Martino Quintavalla, Stefano Bonora, Dario Natali, Andrea Bianco, "Photo controlled deformable mirrors: materials choice and device modeling", Optical Materials Express, Vol. 6, No. 2, (2016)

[11] Martino Quintavalla, Stefano Bonora, Dario Natali, Andrea Bianco, " Zinc selenide-based large aperture photo controlled deformable mirror", Optics Letters, Vol. 41, No. 11, (2016)

[12] Martino Quintavalla, Matteo Baratto, Dario Natali, Stefano Bonora, Chiara Bertarelli, Andrea Bianco, "Fully Organic Photocontrolled Deformable Mirror ", Advanced Optical Materials, Vol. 6, No. 19, (2018)

[13] Edoardo Redaelli, Lorenzo Cabona, Marco Olivieri, Giorgio Pariani, Dario Natali, Andrea Bianco, Frederic Zamkotsian "Photo-controlled deformable mirror based on silicon photoconductor", Proc. SPIE 11448, Adaptive Optics Systems VII, 114485S (2021)

[14] A. Liotard, F. Zamkotsian, "Static and dynamic micro-deformable mirror characterization by phase-shifting and time-averaged interferometry", in Proceedings of the SPIE conference on Astronomical Telescopes and Instrumentation 2004, Proc. SPIE 5494, Glasgow, United Kingdom (2004)

[15] Frédéric Zamkotsian, Giorgio Pariani, Romain Alata, Luca Oggioni, Patrick Lanzoni, Chiara Bertarelli, Andrea Bianco, "The island CGH, a new coding scheme: concept and demonstration", Optics Express, Vol. 27, Issue 19, pp. 26446-26458, (2019)

[16] F. Zamkotsian, P. Lanzoni, E. Grassi, R. Barette, C. Fabron, K. Tangen, L. Valenziano, L. Marchand, L. Duvet "Successful evaluation for space applications of the 2048x1080 DMD," in Proceedings of the SPIE conference on MOEMS 2011, Proc. SPIE 7932, San Francisco, USA (2011) 